\title{
Survival analysis of head and neck cancer: Results from a hospital based cancer registry in southern Karnataka
}

\author{
PU Prakash Saxena ${ }^{\mathrm{a}}$, Bhaskaran Unnikrishnan ${ }^{\mathrm{b}}$, Priya Rathi ${ }^{\mathrm{b}, *}$, Himani Kotian ${ }^{\mathrm{c}}$, \\ Bahgeerathy Reshmi ${ }^{\mathrm{d}}$ \\ ${ }^{a}$ Department of Radiotherapy and Oncology, Kasturba Medical College, Manipal Academy of Higher Education, Mangalore, India \\ ${ }^{\mathrm{b}}$ Department of Community Medicine, Kasturba Medical College, Manipal Academy of Higher Education, Mangalore, India \\ ${ }^{c}$ Department of Community Medicine, Yenepoya Medical College, Mangalore, India \\ ${ }^{\mathrm{d}}$ Department of Health Information Management, School of Allied Health Sciences, Manipal Academy of Higher Education, Manipal, India
}

A R T I C L E I N F O

Keywords:

Cancer registry

Kaplan Meier method

Survival time

TNM staging

\section{Introduction}

Cancer is a major burden of disease worldwide. Each year, millions of people are diagnosed with cancer around the world, and more than half of the patients eventually die from it. ${ }^{1}$ In many countries, cancer ranks as the second most common cause of death following cardiovascular diseases, particularly in developing countries. With significant advances with respect to treatment and prevention of cardiovascular diseases, Cancer has or will soon become the number one killer in many parts of the world. According to the International Agency for Research on Cancer (IARC), a World Health Organization entity, India had 1.8 million people living with cancer (within five years of diagnosis) in 2012. During that year, about a million new cases were recorded, while about $6,83,000$ deaths due to cancer were registered and 32.6 million people were alive after five years of their diagnosis. ${ }^{2-4}$ Five year survival among head and neck cancer patients in developed countries ranged between $0.3 \%$ and $12.6 \%$, whereas in developing country it ranged between $1 \%$ and $4.7 \%$.A study from Mumbai showed a 5 years' survival range between $25 \%$ for cancer tongue to $74 \%$ for lip. ${ }^{5,6}$

Head and neck cancers (HNC) are a heterogeneous group of neoplasms involving mucosal surfaces of the lip, tongue, oral cavity or mouth, oropharynx, nasopharynx, hypopharynx, pharynx not otherwise specified and larynx. ${ }^{7}$ Although there are many histological varieties, the most common head and neck cancers are of squamous cell origin and are referred to as Head and neck squamous cell carcinomas
(HNSCC). ${ }^{8}$

Information on cancer patterns and survival is essential for effective planning of cancer control interventions. Hence, we aimed at studying the clinico-epidemiological profile of HNC patients admitted in a tertiary care hospital from 2013 to 2016 and also to estimate the survival rate in them.

\section{Subjects and methods}

This study was conducted in a medical college teaching hospital, which is a tertiary care multispecialty center. Majority of patients coming to this hospital for cancer related treatments come from poor socio-economic strata due to various government based schemes providing cancer treatment at subsidized rates. This hospital caters to the cancer patients from coastal Karnataka and the north Kerala States. This is a record based retrospective study from our institution. Information was obtained from the records of patients diagnosed during the years of 2013-2016 as suffering from head and neck cancer using a data extraction sheet. Indian Council of Medical Research designated Hospital based cancer registry was established in the year 2015 in this hospital with a dedicated data entry operator and medico social worker. However, the records have been maintained in the institution even prior to the registry.

Case definition: All confirmed and diagnosed Head and Neck cancer cases attending various departments of our hospital during the period

\footnotetext{
* Corresponding author. Department of Community Medicine, Kasturba Medical College, Manipal Academy of Higher Education, Mangalore, 575001, India.

E-mail addresses: pu.saxena@manipal.edu (P. Prakash Saxena), unnikrishnan.b@manipal.edu (B. Unnikrishnan), dr.rathi.priya@gmail.com, priya.rathi@manipal.edu (P. Rathi), himanikotian@gmail.com (H. Kotian).
} 
2013-2016 were included for this study.

Data Collection tool: Data was collected using a data extraction sheet for retrieving the data from record. Patients' sociodemographic characteristics, disease diagnostic details like the stage of cancer, time of detection, previous treatment history, and type of treatment received, and frequency of follow up visit was extracted from records.

\subsection{Data capturing}

Files of confirmed malignancy cases were requisitioned from the medical records department based on the ICD coding. A medical transcriptionist was trained by an oncologist for identifying and capturing the relevant patient details. These patient's social and medical parameters were extracted from the file including the clinical notes and radiological imaging records. These parameters were then used for staging the patient. The American Joint Committee on Cancer (AJCC) 7 th edition was used for the same. Staging and treatment details were verified by an oncologist before entering the data. ${ }^{9}$ Files with incomplete records or no records were not used for analysis.

Patients who could not come for follow-up visits were contacted telephonically to ascertain the current status of the patient's malignancy. In case of no response to the first call, a second phone call was made after a few days. In case of no response to the second phone call, the patient was deemed lost on follow up.

Data analysis: Survival analysis was performed and the predictors for the survival of head and neck cancers were identified. Survival analysis was performed based on following Parameters-Event of interest, Occurrence of death. the starting point was considered from the date of the first diagnosis of cancer and End of follow-up was on 31st May 2017. Kaplan Meier graph was used to report median survival time, if not obtained then mean survival time was reported. Cox hazard model was performed to assess various determinants with the survival of patients. P -value $<0.05$ was considered to be statically significant. All the analysis was performed using SPSS version 22 (IBM Corp. Released, 2013. IBM SPSS Statistics for Windows, Version 22.0. Armonk, NY: IBM Corp.).

\section{Results}

During 2013 to 2016, 454 confirmed head and neck cancer cases were obtained out of which 85 Cases experienced event of interest (death) and 92 cases were alive on 31st May 2017, Rest of the cases could not be traced either due to lack of information in record on the last visit of the patient, or unavailability of the contact number, incorrect contact number. Among 92 cases who were alive, 21 patient's information were obtained through telephonic enquires and rest 71 patient's information were obtained from hospital records.

The maximum follow-up period was 49 months and the mean follow up period was 6.27 months. Out of 454 cases, 365 were males and 85 were females. All the patients belonged to Karnataka and northern part of Kerala. It was seen that the clinico-epidemiological profile of the subset of the patient did not differ from the other patients and it represents the patient's population from where it is being derived (Table 1).

Figs. 1 and 2 depict the survival of HNC patients and Table 2 shows the Cox regression model for the predictors of survival. The interpretation is as follows. There were 70 events of interest (deaths) in males and 15 events in females. The mean survival (median not obtained) for males was 29.35 months as opposed to a mean survival of 21.93 months for females. However, the p-value was not significant $(\mathrm{p}=0.893)$. Similarly, the survival analysis for age category showed a trend towards better survival in the younger age group $(<40$ years and 41-50 years' age group) however the results were not significant $(\mathrm{p}=0.84)$.

Mean survival time (in months) was calculated where the median survival time could not be obtained. Stage 1 patients had the maximal
Table 1

Baseline information and disease details of Head and Neck Cancer patients in the Study.

\begin{tabular}{|c|c|c|}
\hline Characteristics & $\begin{array}{l}\text { All patients (n- } \\
454)\end{array}$ & $\begin{array}{l}\text { Patients included in analysis } \\
(\mathrm{n}=177)\end{array}$ \\
\hline \multicolumn{3}{|l|}{ Age (years) } \\
\hline $18-35$ & $16(3.5)$ & $8(4.5)$ \\
\hline $36-50$ & $127(28.0)$ & $51(28.8)$ \\
\hline$>50$ & $310(68.3)$ & $118(66.7)$ \\
\hline \multicolumn{3}{|l|}{ Gender } \\
\hline Male & $365(80.4)$ & $146(82.5)$ \\
\hline Female & $89(19.6)$ & $31(17.5)$ \\
\hline \multicolumn{3}{|l|}{ Marital status } \\
\hline Married & $453(99.8)$ & $176(99.4)$ \\
\hline Unmarried/Single & $1(0.2)$ & $1(0.6)$ \\
\hline \multicolumn{3}{|l|}{ Religion } \\
\hline Hindu & $407(89.6)$ & $164(92.7)$ \\
\hline Muslim & $24(5.3)$ & $6(3.4)$ \\
\hline Christian & $23(5.1)$ & $7(4.0)$ \\
\hline \multicolumn{3}{|l|}{ Education } \\
\hline Literate & $451(99.3)$ & 175 (98.9) \\
\hline Illiterate & $3(0.7)$ & $2(1.1)$ \\
\hline \multicolumn{3}{|l|}{ Stage (TNM Classification) } \\
\hline 1 & $30(6.6)$ & $14(7.9)$ \\
\hline 2 & $38(8.4)$ & $13(7.3)$ \\
\hline 3 & $92(20.3)$ & $35(19.8)$ \\
\hline $4 a$ & $187(41.2)$ & $66(37.3)$ \\
\hline $4 b$ & $78(17.2)$ & $36(20.3)$ \\
\hline $4 c$ & $29(6.4)$ & $13(7.4)$ \\
\hline \multicolumn{3}{|l|}{ Prior treatment received } \\
\hline Yes & $65(14.3)$ & $29(16.4)$ \\
\hline No & $389(85.7)$ & $148(83.6)$ \\
\hline \multicolumn{3}{|c|}{ Type of treatment in hospital (n-161) } \\
\hline surgery & $51(11.2)$ & 19 (11.8) \\
\hline chemo (palliative) & $47(10.4)$ & $11(6.8)$ \\
\hline radiation (palliative) & $83(18.3)$ & $36(22.4)$ \\
\hline Chemo and radiation & $207(45.6)$ & $72(40.7)$ \\
\hline Surgery + chemo + radiation & $66(14.5)$ & $23(14.3)$ \\
\hline
\end{tabular}

mean survival rate of 40 months. The results were statistically significant $(\mathrm{p}=0.05)$. Survival was highest in a subset of patients who underwent surgery alone as the treatment and lowest for those undergoing palliative radiotherapy alone. The results, however, were not statistically significant. $(\mathrm{p}=0.40)$.

\section{Discussion and Conclusion}

This was a hospital based retrospective record based study of 454 head and neck cancer cases treated during the period of 2013-2016. Head and neck cancer constitute about one-third of all cancer seen at our hospital. This could be attributed to the general trend of HNC in India. ${ }^{10}$

This study shows that male cases were 4 times more compared to female cases and a majority of the patients 310 (68.3\%) were in the $>50$ yrs group which is probably because of the prolonged years of tobacco exposure. This finding is in line with other studies. ${ }^{11}$ In many studies, head and neck cancers are nearly twice as common in men as they are in women and also diagnosed more often in people over age 50 than they are in younger people. ${ }^{12,13}$ The reason for this might be because Indian male is more prone to tobacco chewing, alcohol consumption and smoking compared to that of female and also younger age group are literate and having more awareness of cancer than compared to old age group (Vercelli et al., 2006).

It was also noted that a small proportion of cases belonged to a lower age group. ${ }^{14,15}$ This could be attributed to either the increased prevalence of modifiable risk factors of HNC like tobacco consumption and non-vegetarian diet or genetic predisposition leading to increased incidence of the disease amongst younger population. ${ }^{12,15}$ However, head and neck squamous cell carcinomas can occur in the younger age population ,for example, patient's with p53, p21 mutations (Subapriya 


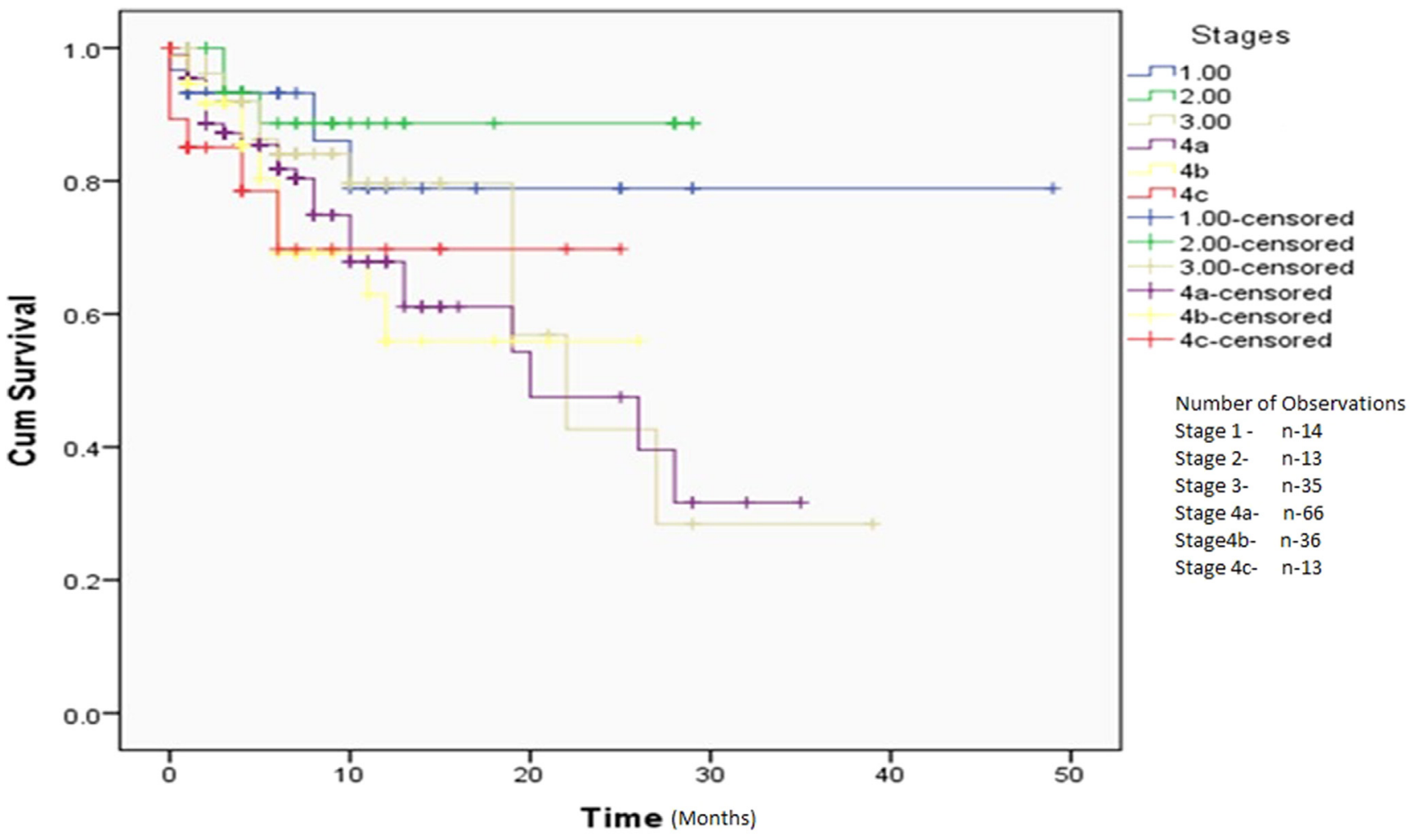

Fig. 1. Survival based on Stage of Head and Neck Cancer.

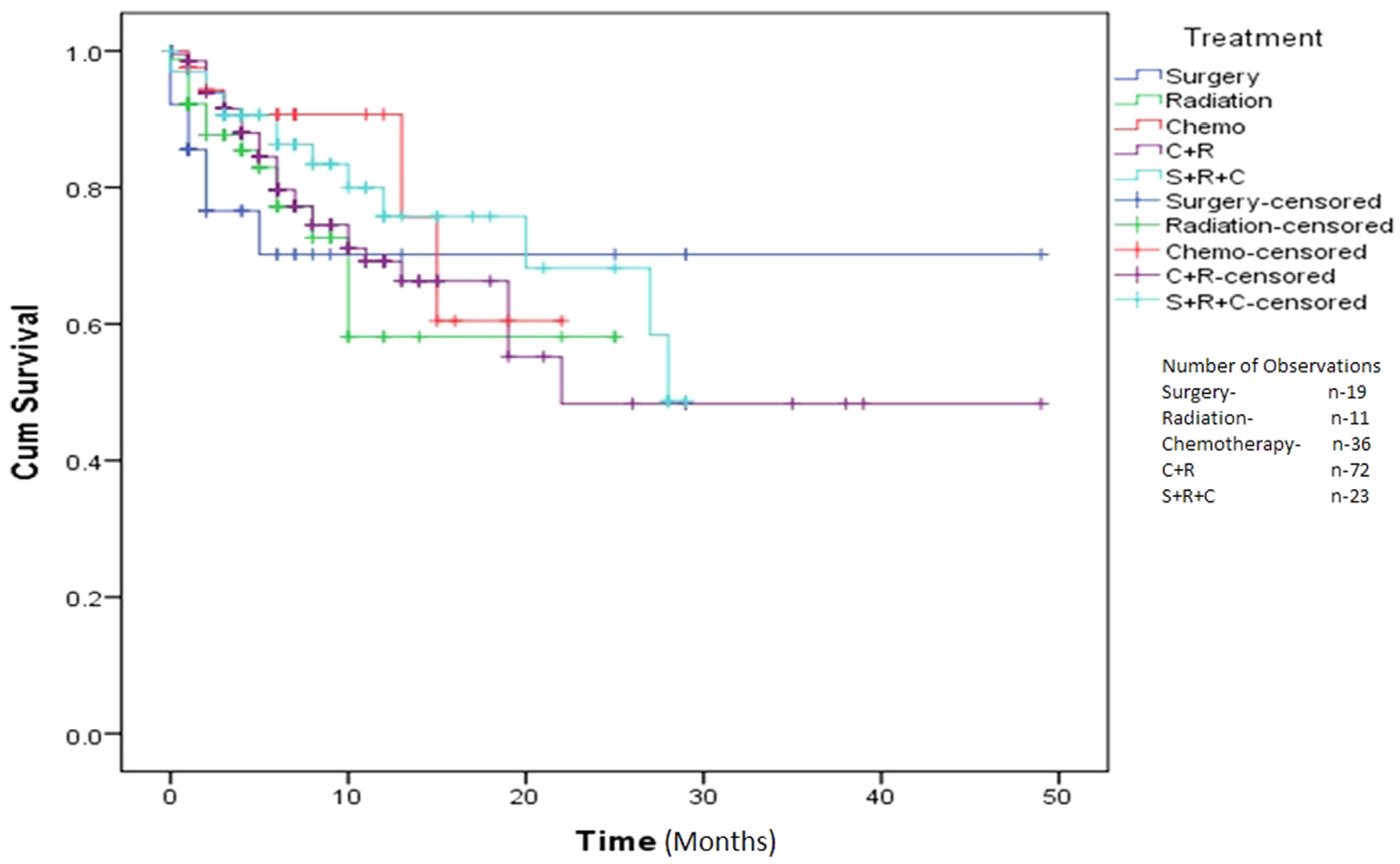

Fig. 2. Survival based on the type of treatment provided to the Head and Neck Cancer Patients. 
Table 2

Association between clinic-social characteristics and disease related factors on survival.

\begin{tabular}{|c|c|c|c|c|}
\hline \multirow[t]{2}{*}{ Characteristics } & \multirow{2}{*}{$\begin{array}{l}\text { Total } \\
\mathrm{N}=177\end{array}$} & \multirow{2}{*}{$\begin{array}{l}\text { survived } \\
\mathrm{N}=94\end{array}$} & \multirow{2}{*}{$\begin{array}{l}\text { Unadjusted OR } \\
\text {-(95\% CI })\end{array}$} & \multirow[t]{2}{*}{$\mathrm{P}$ value } \\
\hline & & & & \\
\hline \multicolumn{5}{|l|}{ Age (years) } \\
\hline $18-35$ & 8 & $5(62.5)$ & REF & 0.8 \\
\hline $36-50$ & 51 & $26(51)$ & $1.6(0.34-7.4)$ & \\
\hline$>50$ & 118 & $63(53.4)$ & $1.4(0.3-6.3)$ & \\
\hline \multicolumn{5}{|l|}{ Gender } \\
\hline Male & 146 & 75 (51.4) & $1.49(0.6-3.3)$ & 0.3 \\
\hline Female & 31 & 19 (61.3) & REF & \\
\hline \multicolumn{5}{|l|}{ Religion } \\
\hline Hindu & 164 & $85(51.8)$ & $2.3(0.4-12.3)$ & 0.4 \\
\hline Muslim & 6 & $4(66.7)$ & $1.2(0.1-13.2)$ & \\
\hline Christian & 7 & $5(71.4)$ & REF & \\
\hline \multicolumn{5}{|l|}{ Stage (TNM Classification) } \\
\hline Early stage (1-2) & 27 & $13(48)$ & $1.2(0.5-2.8)$ & 0.6 \\
\hline Advance stage ( 3 and above) & 150 & $81(54)$ & REF & \\
\hline \multicolumn{5}{|l|}{ Prior treatment received } \\
\hline Yes & 29 & $17(58.6)$ & REF & 0.5 \\
\hline No & 148 & $77(52)$ & $1.3(0.5-2.9)$ & \\
\hline \multicolumn{5}{|c|}{ Type of treatment in hospital (n-161) } \\
\hline surgery & 19 & $10(53)$ & REF & 0.6 \\
\hline chemo & 11 & $7(64)$ & $0.63(.1-2.9)$ & \\
\hline radiation & 36 & $23(64)$ & $0.62(.2-1.9)$ & \\
\hline Chemo and radiation & 72 & $36(50)$ & $1.11(.4-3.0)$ & \\
\hline Surgery + chemo + radiation & 23 & $11(47)$ & $1.21(.3-4.0)$ & \\
\hline
\end{tabular}

et al., 2007). These factors needs to be studies. ${ }^{16}$

The incidence in our study population was more in males than in females. This corresponds with the incidence seen in the subcontinent and also to the fact that tobacco use remains high amongst men. ${ }^{10}$ Majority of our patients were literate (able to both read and write with understanding) (93.3\%) which is higher than the average literacy rate in the state of Karnataka (75.6\%). This could be because of the fact that literate people tend to seek healthcare more often than illiterate ones who often go for more traditional healthcare options. ${ }^{17,18}$

In this study, surgery alone or radiotherapy alone or chemotherapy or their combination remained as the primary treatment. The stage at the time of diagnosis determined the type of treatment the patient received at our institution. Patients in early stages (stage 1\&2) usually underwent surgical resection. Patients with localized or locally advanced disease (stages $3 \& 4 a$ ) either underwent surgery followed by chemo-radiation or were taken up for radical concurrent chemo-radiation. Patients with bulky tumors (Stage 4b) usually underwent palliative radiotherapy and metastatic cases received palliative radiotherapy. Studies on Indian patients have shown that survival of head and neck cancer cases depends largely on the size of the tumor, nodal status, histological variety and stages of disease. ${ }^{19}$

The TNM staging distribution of head and neck cancer in the hospital over three years indicates that a high percentage of cases were seen at an advanced stage of the disease. The present study showed a large percentage of patients present in stage $4 a$ in comparison with reference literature wherein most patients present to hospital in stage 3 of the disease. ${ }^{20}$ This might be because most of the cases are from the poor financial background and they do not have much awareness about the diseases, their ignorance towards self-care, poor access to affordable health care facility, and belief in traditional therapies. ${ }^{21}$

Table 1 also highlights the various treatment modalities offered at our center. Chemo-radiotherapy remains the mainstay of treatment at our center for locally advanced cases where patients are treated for seven weeks along with concurrent cisplatin/carboplatin sensitizers. Although some of the potentially curable cases undergo surgery followed by adjuvant chemo-radiation. Surgery per se remains the standard for early stage head and neck cancers. Palliative therapies like short course radiotherapy and chemotherapy are offered for late stage or metastatic disease or for patients with the poor general condition. These treatment modalities are in line with guidelines which is evidence generated.

As per study objective, we have performed Kaplan Meier Method for study variables and we had to report median survival time. However, for almost all variables we haven't obtained median and we have reported mean survival time, this might be because a majority of the cases were censored cases, Majority of censoring happened $50 \%$ above that of an event happening and highest observed time is also a censored variable.

Patients of younger age group ( $<50$ years) in our study show a trend towards better survival. Younger age group patients usually have a better nutritional status and are able to tolerate radical treatment with curative intent. The low rate of survival among the elderly's likely to be due to poor general health and associated co-morbidities. This pattern of declining survival with age is same as seen previously in Indian population. ${ }^{14,15}$

In our study population male gender, ones diagnosed with early stage cancers $(1,2)$ tended to have a better survival. Patients who take treatment in an institution tend to have better than those who undergo 'cafeteria style' treatment at various healthcare centers. This is because most of the tertiary centers have a tumor board where every case is discussed and a consensus arrived at so as to have best possible results. HNC in a younger patient is usually HPV associated and it's seen that the HPV related HNC are more sensitive to chemotherapy and radiotherapy. ${ }^{22}$ Studies have shown an increase in the incidence of HPV in past two decades. . $^{23,24}$

Also seen from the table is the fact that the patients who underwent treatment like Chemo-radiation or Surgery followed by adjuvant chemo-radiation tend to have a better survival. This highlights the importance of planned combined modality treatment approach in cancer care.

Studies have also shown that married people and those who are literate tend to have a better survival than those who are not. This can be attributable to the fact that married individuals have access to support from their partners and that being educated makes one more inclined towards a healthier life-style and early approach to diagnosis and treatment. Our study population had very few unmarried people but data from similar Indian studies and those from abroad suggest the same. ${ }^{2,15,25}$

An interesting observation from this study is the survival pattern seen based on religion. As per our study group, the unadjusted Odds Ratio for Hindu to Christian was 2.3 with Christians as the reference group. A study conducted by the Bombay cancer registry had also shown similar results, where the observed 5-year survival was 31.7 months for Hindus versus 24.6 months for the Christians. ${ }^{6}$

Our study also highlights the poor survival associated with higher stages at the time of diagnosis, which is similar to existing literature. ${ }^{26}$ This study included patients from Karnataka and Kerala, the finding of the study can be extrapolated to the region.

Since survival analysis requires the status of the patient's mortality, and as it was a retrospective study, a larger proportion of the data had to be censored which is due to the inherent nature of study design. We could consider it as one of the limitations. A cohort study with the newly registered cases could give a better estimate.

This study also highlights the fact that long term follow-up of cancer patients in India is a difficult challenge due to poor socio-economic status and lack of awareness amongst the general public. Most of the patients in our hospital don't come for follow up due to various factors such as the home being far away from the treatment center, costs involved in traveling and staying in the city etc.

\section{Conflicts of interest}

Nil. 


\section{Acknowledgement}

The authors acknowledges the support received from the Indian Council of Medical Research - National Centre for Disease Informatics and Research (NCDIR), Bengaluru.

\section{Appendix A. Supplementary data}

Supplementary data related to this article can be found at https:// doi.org/10.1016/j.cegh.2018.08.007.

\section{References}

1. Ma X, Yu H. Global burden of cancer. Yale J Biol Med. 2006;79:85-94.

2. Sankaranarayanan R, Black RJ, Swaminathan R, Parkin DM. An overview of cancer survival in developing countries. IARC Sci Publ. 1998;145:135-173.

3. Ferlay J, Soerjomataram I, Ervik M, et al. GLOBOCAN 2012 v1.0, Cancer Incidence and Mortality Worldwide: IARC Cancer Base No. 11 Lyon, France. International Agency for Research on Cancer; 2013.

4. Forouzanfar MH, Afshin A, Alexander LT, et al. Global, regional, and national comparative risk assessment of 79 behavioural, environmental and occupational, and metabolic risks or clusters of risks, 1990-2015: a systematic analysis for the Global Burden of Disease Study 2015. Lancet. 388, 1659-1724.

5. Pisani P, Bray F, Parkin DM. Estimates of the world-wide prevalence of cancer for 25 sites in the adult population. Int J Canc. 2002 Jan 1;97(1):72-81

6. Wells MK. Patient adherence to swallowing exercises in head and neck cancer. Curr Opin Otolaryngol Head Neck Surg. 2017;25:175-181.

7. Vigneswaran N, Williams MD. Epidemiological trends in head and neck cancer and aids in diagnosis. Oral Maxillofac Surg Clin. 2014;26(2):123-141.

8. Edge SB, Compton CC. The American Joint Committee on Cancer: the 7th edition of the AJCC cancer staging manual and the future of TNM. Ann Surg Oncol. 2010;17(6):1471-1474.

9. Mishra A, Meherotra R. Head and neck cancer: global burden and regional trends in India. Asian Pac J Cancer Prev APJCP. 2014;15:537-550.

10. Francis CJK. Trends in incidence of head and neck cancers in India. Ann Oncol 1026; 27.
11. Warnakulasuriya S. Global epidemiology of oral and oropharyngeal cancer. Oral Oncol. 2009;45:309-316.

12. Atula S, Grenman R, Laippala P, Syrjanen S. Cancer of the tongue in patients younger than 40 years. A distinct entity? Arch Otolaryngol Head Neck Surg. 1996;122:1313-1319.

13. Vercelli M, Lillini R, Capocaccia R, et al. ELDCARE Working Group. Cancer survival in the elderly: effects of socio-economic factors and health care system features (ELDCARE project). Eur J Canc. 2006 Jan;42(2):234-242.

14. Yeole BB, Jussawalla DJ, Sabnis SD, Sunny L. Survival from breast and cervical cancer in Mumbai (Bombay), India. India IARC Sci Publ. 1998;145:79-87.

15. Subapriya R, Thangavelu A, Mathavan B, Ramachandran CR, Nagini S. Assessment of risk factors for oral squamous cell carcinoma in Chidambaram, Southern India: a case-control study. Eur J Canc Prev. 2014;16:251-256.

16. World Health Organization. General Guidelines for Methodologies on Research and Evaluation of Traditional Medicine. 2000; 2000 80pp.

17. Rudra S, Kalra A, Kumar A, Joe W. Utilization of alternative systems of medicine as health care services in India: evidence on AYUSH care from NSS 2014. PLoS One. 2017;12:1-9.

18. Tuljapurkar V, Dhar H, Mishra A, et al. The Indian scenario of head and neck oncology - challenging the dogmas. South Asian J Cancer. 2016;5:105-110.

19. Fakkhry C. Improved survival of patients with human papillomavirus - positive head and neck squamous cell carcinoma in a prospective clinical trial. J Natl Cancer Inst. 2008;100:261-269.

20. Brenner $\mathrm{H}$. Long-term survival rates of cancer patients achieved by the end of the 20th century: a period analysis. Lancet. 2000;360:1131-1135.

21. Pulte D, Brenner H. Changes in survival in head and neck cancers in the late 20th and early 21st century: a period analysis. Oncology. 2010;15:994-1001.

22. Hammarstedt L, Lindquist D, Dahlstrand H, et al. Human papillomavirus as a risk factor for the increase in incidence of tonsillar cancer. Int $J$ Canc. 2006;119:2620-2623.

23. Gillison ML. Human papillomavirus-related diseases: oropharynx cancers and potential implications for adolescent HPV vaccination. $J$ Adolesc Health 2008;43:S52-S60 2008

24. de Graeff A, de Leeuw JR, Hordijk GJ, et al. Sociodemographic factors and quality of life as prognostic indicators in head and neck cancer. Eur J Canc. 2010;37:332-339.

25. Yeole BB, Sankaranarayanan R, Sunny MSL, Swaminathan R, Parkin DM. Survival from head and neck cancer in Mumbai (Bombay), India. Cancer. 2000;89:437-444.

26. Rao DN, Shroff PD, Chattopadhyay G, Dinshaw KA. Survival analysis of 5595 head and neck cancers results of conventional treatment in a high-risk population. $\mathrm{BJC}(\mathrm{Br}$ J Cancer). 1998;77:1514-1518. 